\title{
3D Prostate Surface Detection from Ultrasound Images Based on Level Set Method
}

\author{
Shao Fan $^{1} \quad$ Ling Keck Voon ${ }^{2} \quad \mathrm{Ng} \mathrm{Wan} \mathrm{Sing}^{3}$ \\ ${ }^{1}$ School of Electrical \& Electronic Engineering, P149624243@ntu.edu.sg \\ ${ }^{2}$ School of Electrical \& Electronic Engineering, ekvling@,ntu.edu.sg \\ ${ }^{3}$ School of Mechanical \& Production Engineering, MWSNG@,ntu.edu.sg \\ CIMIL - Computer Integrated Medical Intervention Laboratory
}

Nanyang Technological University, 50, Nanyang Avenue, Singapore 639798

http://mrcas.mpe.ntu.edu.sg/

\begin{abstract}
Accurate detection of prostate boundaries is required in many diagnostic and treatment procedures for prostate diseases. In this paper, a new approach based on level set method to perform 3D prostate surface detection from transrectal ultrasound (TRUS) images is presented. Contrary to many other deformable models, level set method offers several advantages such as minimal need for user input, flexible topology, and straightforward extension to 3D. However, it is subject to "boundary leaking" problem for ultrasound image segmentation due to the poor image quality. In this work, we first develop a fast discrimination method to extract the prostate region, then this region information, instead of the spatial image gradient, is incorporated into the level set method to remedy the "boundary leaking" problem. Various experimental results show the effectiveness of the proposed method.
\end{abstract}

\section{Introduction}

Prostate diseases are common in adult and elderly men. Typical symptoms are benign prostatic hyperplasia (BPH) and prostate cancer. With the number of men seeking medical care for prostate disease rising steadily, the need of a fast and accurate prostate boundary detection and volume estimation tool increases correspondingly. Currently, boundary detection and volume measurement are made manually, which is arduous and user dependent. A possible solution is to improve the efficiency by automating the boundary detection and volume estimation process with minimal manual involvement. This paper presents a new approach based on level set method [1] to semi-automatically detect the prostate surface from 3D transrectal ultrasound (TRUS) images.

There have been a number of works so far on automatic segmentation of prostate from ultrasound images. A straightforward strategy is using edge detectors, such as Minimum/Maximum filter [2], derivative edge detectors [3, 4], sticks and weak 
membrane fitting [5], to identify all the edges in the image then followed by edge selection and linking to outline the prostate boundary. However, due to the poor quality of ultrasound images, such kind of methods usually leads both to spurious boundaries in highly textured areas and to missed boundaries where prostate boundary is not well-delineated.

Richard et al. [6] used texture features to segment 2D images of the prostate gland into prostate and non-prostate regions for forming a $3 \mathrm{D}$ image of the prostate from a set of parallel 2D images. Although some progress has been made, they acknowledged that the effect of using texture information is marginal.

More efficient way is using deformable models, such as discrete dynamic contour (DDC) [7] and 3D deformable surface [8]. However, the success of their approaches is dependent on the careful initialization of the contour or surface, which requires the user to select points on the prostate boundary. In [9], wavelet-based techniques have been used to attempt to address this problem.

Some researchers employed neural networks [10] and feature modeling [11] to segment the prostate from TRUS images. As reported in their work, these methods have good accuracy and robustness. However, neural networks requires extensive teaching sets so that the speed is very slow and feature modeling is only suitable for some particular shape-based prostate images.

In this work, we develop a new approach based on level set method to automatically detect the prostate surface from 3D TRUS images. We first develop a fast discrimination method to extract the prostate region. Then this region information is incorporated into the level-set method instead of the spatial image gradient. In the following, we first give a brief description of our method and then we discuss the results.

\section{Methods}

While deformable models, such as Snakes [12], Fourier Surface [13] and Free-Form Deformation (FFD) [14] have been widely used in medical imaging applications, they have severe limitations: they are unable to handle complex geometry and changing topology without additional machinery, and complex implementation in 3D. To overcome these difficulties, the level set method has been proposed [1]. In this approach, a $2 \mathrm{D}$ curve $C$ is represented by a $3 \mathrm{D}$ function $\psi$. The value of the $3 \mathrm{D}$ function at point $p$ is defined as a distance $d$ from $p$ to $C$ according to equation (1):

$$
\psi(p, t=0)= \pm d
$$

where $p \in \mathfrak{R}^{2}$ are points in the image space, and the plus (minus) sign is chosen if the point $p$ is outside (inside) the $2 \mathrm{D}$ curve $C(t=0)$. In this manner, the $2 \mathrm{D}$ curve is represented by the zero level set $C(t)=\{p \mid \psi(p, t)=0\}$ of the level set function $\psi$. The level set method then evolves the 3D function $\psi(p, t)$ that contains the embedded motion of $C(t)$ instead of the original 2D curve. The evolution of the 3D function $\psi$ can be expressed by means of a partial differential equation (PDE) as 


$$
\frac{\partial \psi(p, t)}{\partial t}+F|\nabla \psi|=0
$$

with a given initial condition $\psi(p, t=0)$, where $\nabla \psi$ denotes the gradient of $\psi$ with respect to the spatial coordinates and $F$ is the evolving speed.

For numerical solution of equation (2), it is necessary to perform discretization in both space and time. For this purpose we can discretize space coordinates using a uniform mesh of spacing $h$, with grid nodes denoted by indices $i j$. Let $\psi_{i j}^{n}$ be the approximation to the solution $\psi(i h, j h, n \Delta t)$, where $\Delta t$ is the time step. The expression for $\psi_{i j}^{n+1}$ can be derived using the forward definite difference method:

$$
\psi_{i j}^{n+1}=\psi_{i j}^{n}-\Delta t F\left|\nabla \psi_{i j}^{n}\right| .
$$

Let $K$ be the mean curvature of the evolving front, then $K$ can be easily obtained from the divergence of the gradient of the unit vector to front, i.e.,

$$
K=\nabla \cdot \frac{\nabla \psi}{|\nabla \psi|}=\frac{\psi_{x x} \psi_{y}^{2}-2 \psi_{x} \psi_{y} \psi_{x y}+\psi_{y y} \psi_{x}^{2}}{\left(\psi_{x}^{2}+\psi_{y}^{2}\right)^{3 / 2}}
$$

As reported in [1], the speed term $F$ depends on the curvature $K$ and is separated into a constant term $F_{0}$ and the remainder $F_{1}(K)$, that is

$$
F(K)=F_{0}+F_{1}(K) .
$$

The constant term $F_{0}$ causes the model to seek object boundaries and the curvature component $F_{1}$ controls the regularity of the deforming shape. In practice, speed

$$
F(K)=1-\varepsilon K
$$

is commonly used, where $\varepsilon$ is the entropy condition which regulates the smoothness of the curve and $\varepsilon$ must be greater than zero [1].

To ensure the propagating curve front will stop in the vicinity of desired object boundary, $F$ is proposed to be pre-multiplied with an image dependent quantity $k_{I}[1]$,

$$
k_{I}(x, y)=\frac{1}{1+\left|\nabla G_{\sigma} * I(x, y)\right|^{p}}, \quad p=1,2
$$

where $G_{\sigma}{ }^{*} I$ denotes image convolved with the Gaussian smoothing filter whose characteristic width is $\sigma . k_{I}$ has values close to zero in regions of high image gradient (for example, possible edges) and close to unity in regions with relatively constant intensity. Clearly, the key task of this level set method is to design an appropriate speed function $F$ which can drive the evolving front to the desired boundary.

Unlike other deformable models, extending the level set method to 3D is straightforward [1]. In that case, $\psi$ is a $4 \mathrm{D}$ scalar function, $\psi: \mathfrak{R}^{3} \times \mathfrak{R}^{+} \rightarrow \mathfrak{R}$, which evolves over time and its mean curvature can be expressed as: 


$$
K=\frac{\psi_{x x}\left(\psi_{y}^{2}+\psi_{z}^{2}\right)+\psi_{y y}\left(\psi_{x}^{2}+\psi_{z}^{2}\right)+\psi_{z z}\left(\psi_{x}^{2}+\psi_{y}^{2}\right)-2\left(\psi_{x y} \psi_{x} \psi_{y}+\psi_{x z} \psi_{x} \psi_{z}+\psi_{y z} \psi_{y} \psi_{z}\right)}{\left(\psi_{x}^{2}+\psi_{y}^{2}+\psi_{z}^{2}\right)^{3 / 2}} .
$$

Due to the intrinsic features of ultrasound images, such as noises, speckles, shadowing and low contrast, the boundary feature of the object is usually not salient enough and the image gradient information is weak. These cause the "boundary leaking" problem when we apply the level set method to detect the 3D prostate surface as shown in Fig. 1. In this test, $F=1-0.375 K$, the Gaussian smoothing filter characteristic width $\sigma$ is set to 0.75 and a Gaussian kernel width 5 has been used.

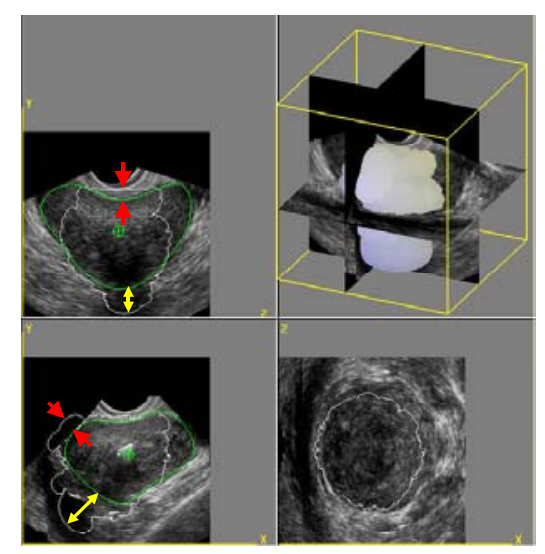

Fig. 1. An example of "boundary leaking" problem of level set method. White curve: the detected boundary of the level set method; Green curve: the manually outlined boundary. Yellow arrow: the boundary leaking caused by shadowing; Red arrow: the boundary leaking caused by low contrast.

Motivated by the region-based strategy for active contour models as reported in $[15,16]$, we integrated the region information instead of the image gradient into the level set method to improve the model performance. First we develop a fast discrimination method to extract the prostate region according to the intensity likelihood as:

$$
R(x, y, z)= \begin{cases}0 & \text { if } 2<\left[\max \left(I_{n}\right)-\min \left(I_{n}\right)\right]<\text { givenThreshold } \\ 1 & \text { else }\end{cases}
$$

where $\max \left(I_{n}\right)$ and $\min \left(I_{n}\right)$ stand for maximal intensity and minimal intensity respectively in a $n^{3}$ slide window. We take the lower bound as 2 to exclude the black background in the images. In our algorithms, we take $n$ as 5 empirically and the givenThreshold, set by the user, could be of different values for different images, we denote it $R_{T}$ for simplicity in the following sections. 
This function is then incorporated into the level set method and forms a new speed function as:

$$
F_{\text {new }}=F_{0} \cdot(1-R)-\varepsilon K
$$

Now $F_{n e w}$ is not related to image gradient any more, it only depends on the prostate region and evolving front curvature. Consequently, $F_{\text {new }}$ has the following properties:

1. $R=0$, then

$$
F_{n e w}=F_{0}-\varepsilon K
$$

This means inside the prostate region, the evolving front will deform according to the speed as expressed in equation (6), no image constraints are included.

2. $R=1$, then

$$
F_{n e w}=-\varepsilon K
$$

This means outside the prostate region, the evolving front will shrink.

Therefore, the interaction of these two properties makes the evolving front eventually attracted to the desired boundary. In our algorithm, $F_{0}=\varepsilon=2$ is selected empirically and the Gaussian filter is replaced by the median filter for the latter's good features of removing speckle noise efficiently and at the same time preserving boundary information.

In this work, all the input images to our algorithm are collected from Singapore Gleneagles Hospital by using Voluson 530D.

\section{Experimental Results}

We applied the proposed method to 8 3D TRUS images to detect the prostate surface. Figure 2 shows one of the results detected by our new approach. The 3D image size is $256 \times 256 \times 256$. The initial and final 3D shapes are shown in the right and the slices shown as transverse view, sagittal view and coronal view respectively are selected by the cutting plane as shown in (f). For this 3D image, we set $R_{T}$ to 48 .

To validate the effectiveness of our new approach, we first compare the above result with the manually outlined contours (drawn by an expert from Singapore General Hospital) in cross-sectional images in figure 3. It can be seen there is good agreement between the detected contours and the manual contours except some divergence in the right sides of sagittal view and coronal view. ${ }^{1}$ The new method is then applied to other patients' images. One of the tests is shown in figure 4, where the image size is $250 \times 174 \times 236$ and the $R_{T}$ is set to 32 . Although the image quality is quite poor, our new approach successfully detected most of the desired boundaries.

${ }^{1}$ The validation presented in this paper is purely subjective. Quantitative assessment method is still under development as addressed in section 4 . 


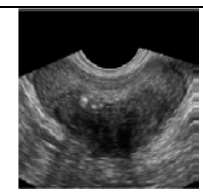

(1)

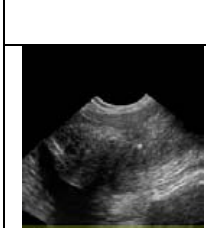

(1)

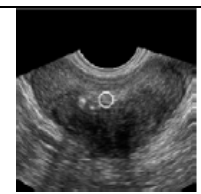

(2)

(a)

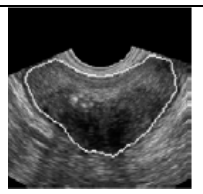

(3)

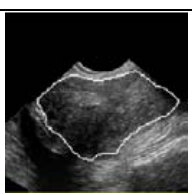

(3)

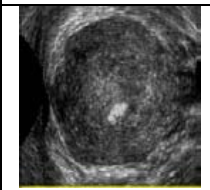

(1) (b)

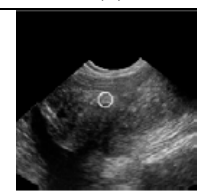

(2)

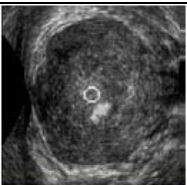

(2)

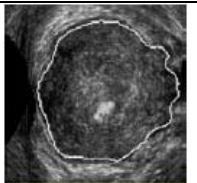

(3)

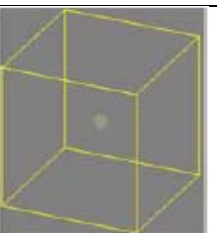

(d)

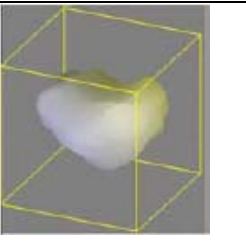

(e)

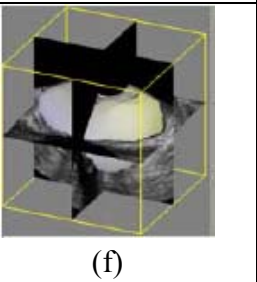

Fig. 2. 3D prostate surface and its cross-sectional contours detected by our new approach. (a) Transverse view, (b) Sagittal view, (c) Coronal view [(1) A slice of the original image, (2) Slice with the initial contour overlaid, (3) Slice with the final contour overlaid.], (d) Initial surface, (e) Final surface, (f) Final surface with cutting planes.

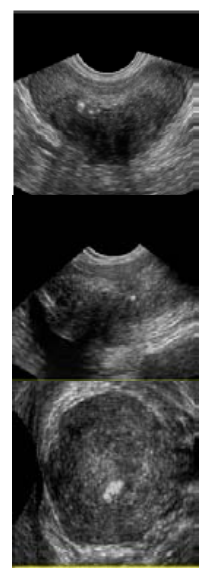

(a)

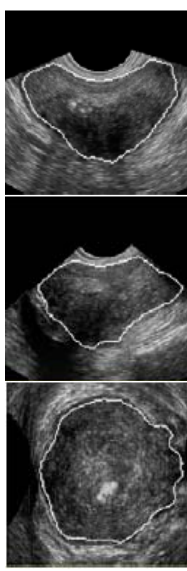

(b)

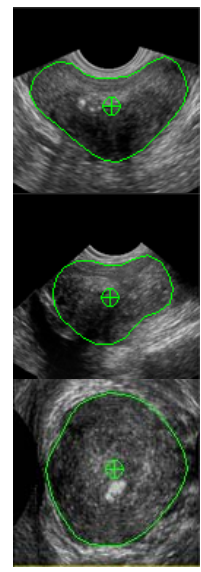

(c)

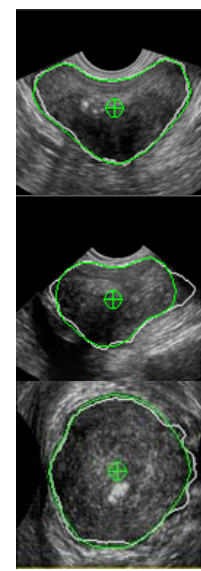

(d)

Fig. 3. The comparison between detected contours and manual contours in $2 \mathrm{D}$ images. (a) Cross-sectional slices, (b) Detected contours, (c) Manual contours, (d) contour comparison. 


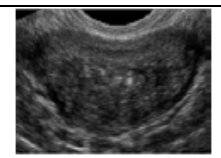

(1)

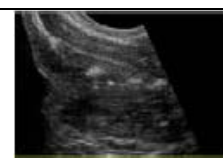

(1)

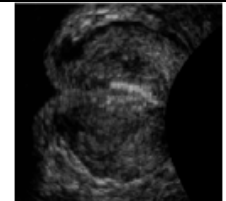

(1)

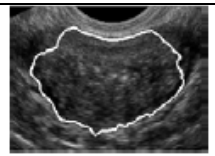

(2)

(a)

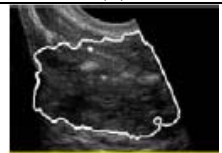

(2)

(b)

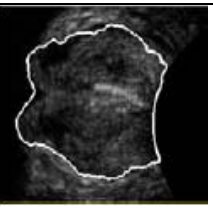

(2)

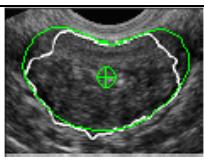

(3)

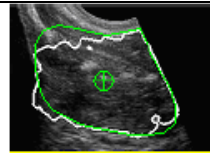

(3)

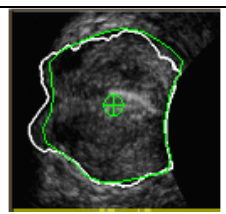

(3)

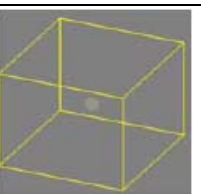

(d)

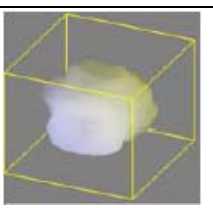

(e)

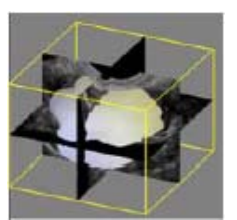

(f)

(c)

Fig. 4. An example of algorithm tests on different patients' images. (a) Transverse view, (b) Sagittal view, (c) Coronal view [(1) A slice of the original image, (2) Final detected contour, (3) Final detected contour with manual contour overlaid.], (d) Initial surface, (e) Final surface, (f) Final surface with cutting planes.

\section{Discussion}

In our algorithm, parameters such as $F_{0}, \varepsilon$ and slide window size $n$ for region discrimination are pre-determined experimentally and keep unchanged in various implementations over different images. In these implementations, we take a small sphere as the initial surface put at the image center to start the detection procedure, thus only one parameter $R_{T}$ should be set by users and consequently the human involvement is minimized. However, because the prostate region discrimination in our algorithm is still simple and coarse, the final detected results are sensitive to the choice of $R_{T}$. This problem might be solved by including the statistical information of the image intensity distribution. Therefore, an important future work on this research is to investigate the image intensity distributions. Besides, we also plan to introduce some a priori knowledge of the prostate shape to constrain the surface deformation to improve the algorithm accuracy [17]. Assessment method should be included too to evaluate the experimental results quantitatively. 


\section{References}

1. Sethian, J.A.: Level Set Methods and Fast Marching Methods - Evolving Interfaces in Geometry, Fluid Mechanics, Computer Vision, and Materials Science. Cambridge University Press (1996)

2. Aarnink, R.G., Pathak, S.D., de la Rosette, J.J.M.C.H., Debruyne, F.M.J., Kim, Y., Wijkstra, H.: Edge detection in prostatic ultrasound images using integrated edge maps. Ultrasonics 36 : 635-642, 1998

3. Lee J.Y., Chen C.H., Hsien H.B., Yang D.L., Sun Y.N.: 3D reconstruction of prostate from transrectal ultrasound images. in Proceedings of the International Conference \& Exhibition on Electronic Measurement \& Instrumentation, Shanghai, China (1995) 19-26

4. Chen, C.H., Lee, J.Y., Yang, W.H., Chang, C.M., Sun, Y.N.: Segmentation and reconstruction of prostate from transrectal ultrasound images. Biomed.Eng. Appl. Basis. Biomm., 8(3) : 287-292, 1996

5. Pathak, S.D., Chalana, V., Haynor, D.R., Kim, Y.: Edge-Guided Boundary Delineation in Prostate Ultrasound Images. IEEE Transactions on Medical Imaging, 19(12): 1211-1219, 2000

6. Richard, W.D., Keen, C.G.: Automated texture-based segmentation of ultrasound images of the prostate. Comput. Med. Imag. Graph., 20(3) : 131-140, 1996

7. Ladak H.M., Mao Fei, Wang Yunqiu, Downey D.B., Steinman D.A., Fenster A.: Prostate segmentation from 2D ultrasound images. Med. Phys., 27(8) : 1777-1788, 2000

8. Ghanei, A., Soltanian-Zadeh, H., Ratkewicz, A., Fang-Fang Yin: A three-dimensional deformable model for segmentation of human prostate from ultrasound images. Med. Phys., 28(10): 2147-2153, 2001

9. Knoll, C.J., Alcaniz-Raya, M.L., Monserrat, C., Grau Colomer, V., Juan, M.: Multiresolution segmentation of medical images using shape restricted snakes. SPIE Med. Imag., $3661:$ 222-233, 1999

10. Prater, J.S., Richard, W.D.: Segmenting ultrasound images of the prostate using neural networks. Ultrason. Imag., 14 : 159-185, 1992

11. Wu R.Y., Ling K.V., Ng W. S.: Automatic Prostate Boundary Recognition in Sonographic Images Using Feature Model and Genetic Algorithm. Journal of Ultrasound in Medicine, 19(11): 771-782, 2000

12. Kass, M., Witkin, A., Terzopoulos, D.: Snakes: active contour models. International Journal of Computer Vision, 1(4) : 312-331, 1988

13. Staib, L.H., Duncan, J.S.: Model-based deformable surface finding for medical images. IEEE Transactions on Medical Imaging, 15(5) : 720-731, 1996

14. Sederberg, T.W., Parry, S.R.: Free-form deformation of solid geometric models. Computer Graphics (Proceedings of SIGGRAPH), 20(4) : 151-160, 1986

15. Ronfard, R.: Region-based strategies for active contour models. International Journal of Computer Vision, 13(2): 229-251, 1994.

16. Poon, C.S., Braun, M.: Image segmentation by a deformable contour model incorporating region analysis. Phys. Med. Biol., 42(9) : 1833-1841, 1997

17. Leventon M., Faugeraus O., Grimson W., Wells W.: Level set based segmentation with intensity and curvature priors. in Workshop on Mathematical Methods in Biomedical Image Analysis Proceedings (2000) 4-11 